\title{
BMJ Open Multicentre randomised study of the effect and experience of an early inhome programme (PreHomeCare) for preterm infants using video consultation and smartphone applications compared with inhospital consultations: protocol of the PreHomeCare study
}

\author{
Mai-Britt Hägi-Pedersen, ${ }^{1,2}$ Annelise Norlyk, ${ }^{1,3}$ Ram Dessau, ${ }^{4}$ Hristo Stanchev, ${ }^{2}$ \\ Hanne Kronborg ${ }^{1}$
}

To cite: Hägi-Pedersen $M-B$, Norlyk A, Dessau R, et al. Multicentre randomised study of the effect and experience of an early inhome programme (PreHomeCare) for preterm infants using video consultation and smartphone applications compared with inhospital consultations: protocol of the PreHomeCare study. BMJ Open 2017;7:e013024. doi:10.1136/bmjopen-2016013024

- Prepublication history for this paper is available online. To view these files please visit the journal online (http://dx.doi.org/10.1136/ bmjopen-2016-013024).

Received 13 June 2016 Revised 18 October 2016 Accepted 16 January 2017

CrossMark

For numbered affiliations see end of article.

Correspondence to Mai-Britt Hägi-Pedersen; maiha@regionsjaelland.dk

\section{ABSTRACT}

Introduction: Although premature infants and their parents are discharged earlier to inhomecare programmes, how to optimally support parents during this transition remains unknown. The aim of this study is to compare the effects of early inhomecare (PreHomeCare) including video consultations and mobile applications with those of inhospital consultations regarding breast feeding, parental confidence and parent-infant interactions.

Methods and analysis: A randomised controlled intervention study will be conducted in four neonatal departments offering PreHomeCare (ie, premature infant inhomecare) in Denmark. Parents of hospitalised premature infants who fulfil the inclusion criteria for PreHomeCare will be randomised during hospitalisation to either the intervention $(n=80)$ or control group $(\mathrm{n}=80)$ using 1:1 block randomisation. During PreHomeCare, the intervention group will receive a smartphone application with a video system and an infant scale, and the control group will receive usual care (ie, hospital consultations). Additionally, both groups will have planned nurse consultations two to three times a week: the intervention group through video consultations and the control group through inhospital consultations. Data collection will occur at inclusion/baseline, at the end of PreHomeCare and 1 month after discharge using questionnaires and hospital records. The primary outcome is the proportion of exclusively breastfed infants 1 month after discharge/end of PreHomeCare, the secondary outcomes are parent-infant interactions measured by the Mother and baby interaction scale and family confidence in caring for infants measured by the Karitane Parenting Confidence Scale. The process evaluation will consist of two qualitative studies: a field study and an interview study. Data collection will initially involve field observations of three scheduled video consultations with six families from the intervention group. These families will also be interviewed 1 month after PreHomeCare has ended. Ethics and dissemination: The project has been approved by the Regional Ethics Committee and the Danish Data Protection Agency.

Trial registration number: NCT02581800.

\section{BACKGROUND}

Preterm birth rates have been increasing in almost all countries worldwide, ${ }^{1}$ and $\sim 15$ million infants around the world are born prematurely each year. Premature birth is defined as a delivery that occurs before a gestation age (GA) of 37 weeks. ${ }^{2}$ In Denmark, $\sim 6.4 \%$ of all live infants are born prematurely. ${ }^{3}$ Premature infants are characterised by organ underdevelopment, which increases the need for treatment associated with breathing, circulatory and gastrointestinal function, temperature regulation and infections; in most cases, these needs require admission to the neonatal ward. $^{2}$ While health professionals care for the infant (depending on the severity of the infants' condition), parents often experience anxiety, depression, decreased parenting confidence and selfefficacy and impaired parent-child interactions related to feelings resulting from the 
premature infant's condition. ${ }^{4}$ Parental anxiety related to uncertainty about their infant's health has been shown to affect infant development and growth and perceptions of future infant vulnerability. ${ }^{4}$ Furthermore, parental anxiety seems to be a significant predictor of cognitive and behavioural outcomes in later life. ${ }^{5}$

Breast feeding or bottle nutrition is initiated during hospitalisations, and as the premature infant grows and develops, the parents gradually assume responsibility for the provision of primary care. It is important for premature infants to receive breast milk because it provides pronounced health benefits. ${ }^{6}$ Mothers are encouraged to express breast milk approximately every third hour to feed their infant in order to stimulate the breast and prepare for when the infant is ready to breastfeed. Despite these recommendations, at discharge, premature infants are not breast fed to the same extent as term infants. Among other factors, a low GA, mother's lack of privacy during breast feeding, mother's level of stress and the lack of use of baby-friendly initiatives in neonatal wards seem to limit breast feeding success. ${ }^{6} \mathrm{~A}$ previous study showed positive effects of test weighing on establishing earlier exclusive breast feeding. ${ }^{7}$ Additionally, a newly published study found that the proportion of breast feeding decreases from $68 \%$ at discharge to $\sim 40 \%$ around 1-month post discharge from the neonatal ward. ${ }^{8}$ A Cochrane review further demonstrated that any form of extra support can increase the duration of exclusive breast feeding in term infants. ${ }^{9}$ These findings indicate that there may be a need to create an environment that supports maternal breast feeding of premature infants. In a recent study, parents of premature infants reported that they did not feel that the infant was theirs before returning home. ${ }^{10}$

Towards the end of their hospital stay, most premature infants will be physiologically stable, although some will still require tube feeding. ${ }^{11}$ At this point, in most hospitals in Denmark, families are provided the opportunity to participate in early inhomecare programmes to bring their infant home as well as to receive relevant education. These programmes are designed to bridge the gap in the transition from hospital to home and provide parents and infants the ability to be alone and become familiarised with each other outside of the hospital setting. ${ }^{10}{ }^{12}$ Early inhomecare with tube feeding performed by parents ${ }^{10} 11{ }^{13-16}$ is becoming the standard of care in most Danish hospitals. Qualitative studies have described how the transition to early inhomecare is a difficult experience for new parents, particularly in the first weeks and months after discharge. ${ }^{2}{ }^{4}$ Although parents in this phase have been looking forward to returning home, they often feel insecure and concerned about their infant's health and well-being. ${ }^{17}$ At the same time, they want to have control over the situation and function as normal parents regarding their infant. ${ }^{18}$ In a recent study, parents received knowledge and education ${ }^{4}$ to accommodate this difficult transition. The families expressed the importance of being assigned to the same nurse throughout the entire process ${ }^{2}$ and of receiving unidirectional and additional guidance. Early inhome programmes can address parent-child interactions, ${ }^{19}$ which have been shown to have a significant effect on infant development, ${ }^{4}$ and could also support mothers in successful breast feeding.

Early inhomecare programmes have previously been followed by support ${ }^{20}$ from home visits and/or hospital consultations two to three times a week or by the use of telemedicine communication. ${ }^{16}{ }^{21}$ The effects of home visits and/or hospital consultation support include the prolonged provision of breast milk, ${ }^{22}$ a lower risk of infections during tube feeding ${ }^{20}$ and higher parental satisfaction; ${ }^{14}$ furthermore, early inhomecare for premature infants results in fewer readmissions, less unscheduled support and shorter lengths of hospitalisation. ${ }^{14}$ A new Cochrane review and a randomised study on these programmes were not able to find significant differences in exclusive or partial breast feeding. ${ }^{11} 20$ Moreover, no differences in parental anxiety or confidence were found; however, mothers in early inhomecare programmes felt better prepared to take care of their infants. ${ }^{11}$ Although home visit support shows promising results, it requires comprehensive nurse resources and can therefore be an expensive and resourcedemanding approach. ${ }^{23}$ To date, no studies have offered round-the-clock home visit support. A randomised controlled effect study from Sweden that used less resourcedemanding video consultations showed that families welcomed this option and that the need for home visits was reduced. ${ }^{16}{ }^{24}$ These findings indicate that telemedicine may be a viable option to address the need for support in the early inhomecare period. Furthermore, using an application to collect daily data has great potential in telemedicine. ${ }^{16}$ The use of technology provides parents the opportunity to be at home and still be in close contact with hospital staff, ${ }^{16} 21{ }^{23-26}$ and it gives hospital staff the opportunity to provide equal care to all parents regardless of their distance from the hospital. The concerns raised about the use of technology have been that it affects the communication between parents and nurses ${ }^{27}$ and that the occurrence of frozen screens may affect video communications. ${ }^{28}$ Telemedicine is a very new and unexplored field in the neonatal setting, and there is a lack of knowledge of its effects on breast feeding, parental confidence and parent-infant interactions as well as a lack of experience with the use of video consultations and smartphone applications. The aim of this study is to examine the effect (1) and evaluate the process (2) of early inhomecare (PreHomeCare) with video consultations and mobile applications compared with the effect of inhospital consultations. The hypothesis is that the intervention will positively influence the continuation of breast feeding by providing ${ }^{1}$ easily accessible knowledge about breast feeding (application), ${ }^{2}$ available guidance at all hours from a nurse (video consultation) and $^{3}$ home weighing of the infant (weight). Effect study (1): The objective is to test the 
effects of video consultations and the mobile application on the proportion of exclusive breastfeeding mothers, parent-infant interactions and parental confidence with premature infants after the PreHomeCare programme. Process evaluation studies (2): The objectives are to explore nurses' communication (regarding nursing practice, dialogue and information) during the parent-nurse interactions in the video consultations and to explore how parents of premature infants experience PreHomeCare and the use of technology.

\section{PREHOMECARE STUDY \\ Method}

Mixed methods study design

The effects and process of the project will be evaluated using the framework outlined by the intervention mapping approach. ${ }^{29}$ The study will apply a mixed methods design comprising a randomised controlled intervention study with two parallel arms for the evaluation of effects, targeting the primary study population and two qualitative studies for the process evaluation of the intervention: a field study and an interview study, primarily targeting the secondary study population of nurses involved in the intervention.

The primary study population of early discharged parents with premature infants will be randomised into two groups: the intervention group, which will receive the smartphone application and video consultations in the PreHomeCare programme, and a control group, which will receive usual care (hospital consultations) in the PreHomeCare programme (see figure 1).

\section{Setting}

There are 19 neonatal wards in Denmark, and this study is being conducted in four of them (in different geographical parts of Denmark). Study inclusion began in November 2015. These wards all receive premature infants from GA week 27/28 and correspond to a level IIIa ward. ${ }^{30}$

PreHomeCare: The care in these neonatal wards is provided by registered nurses (RN) who are trained in caring for premature infants. All nurses can participate in the early inhomecare programme, as all are familiar with and trained in this type of care; however, 3-4 nurses on each ward have special interests in early inhomecare. These nurses are the most experienced in this field and work in practice and in experience sharing and will thus serve as the nurses responsible for the project. The wards assign the families a contact nurse who follow the family through the admission and early inhomecare.

The criteria to participate in early inhomecare are that the infant has a GA $\geq 34+0$ at the start of inhomecare, does not have apnoea, maintains a normal body temperature, gains weight, receives tube feeding, has started breast feeding/bottle nutrition and does not need medical treatment. The goals of early inhomecare are to strengthen the parent-child relationship, support the parents' skills, ease the transition to home and support mothers in practicing exclusive breast feeding.

When the family returns home in early inhomecare (usual care), they attend 2-3 planned visits to the hospital to talk with a nurse, primarily the contact nurse. The content of this discussion pertains to the nutrition plan, infant's actual weight, bottle/breast feeding progression, family life, infant's general well-being, expression of breast milk, use of nipple shields and tube feeding, among other topics.

\section{Study status}

As of September, 50 infants/parents from the participating neonatal wards had been enrolled in the study. We expect to finish enrolment in 2018.

\section{Inclusion and exclusion criteria}

Parents who have hospitalised infants born before 37 weeks' gestation who fulfil the criteria for early inhomecare, who speak Danish or English, understand the Danish text in the application and have Wi-Fi/LTE/ HSDPA in their home (programme phones provide LTE/HSDPA) will be invited to participate.

The exclusion criteria are infants who do not meet the criteria for early inhomecare or parents who require additional parent-infant observations regarding attachment or eye contact or have low parenting skills based on an individual assessment of the nurses or doctors.

\section{Plan for randomisation}

The parents will be informed about the PreHomeCare programme by the nurses on admission to the neonatal ward. When the infant starts breast feeding or bottle feeding, the parents will be informed verbally and in writing about the project if they are interested in going home. After obtaining written consent from the parents and consent for their infant's participation, they will be randomised to either the intervention or control group using fixed block randomisation in a 1:1 ratio per site (at the individual level) using a website-generated randomisation procedure. At inclusion, the nurses will have access to the website to obtain the randomisation result. The inclusion and exclusion of participants will follow the Consolidated Standards of Reporting Trials (CONSORT) statement, ${ }^{31}$ see figure 2.

\section{Programme implementation and monitoring procedures}

The secondary study population primarily consists of nurses. To ensure a standardised implementation of the programme, the protocols will outline how the programme should be delivered. An instruction manual for the nurses has been developed in Danish ${ }^{32}$ to ensure that the application is easy to understand and use. The manual contains instructions and pictures of the application to guide the user. All four participating wards have three or four project nurses (the nurses with a special interest in early inhomecare) who have received training 
Figure 1 Project overview.
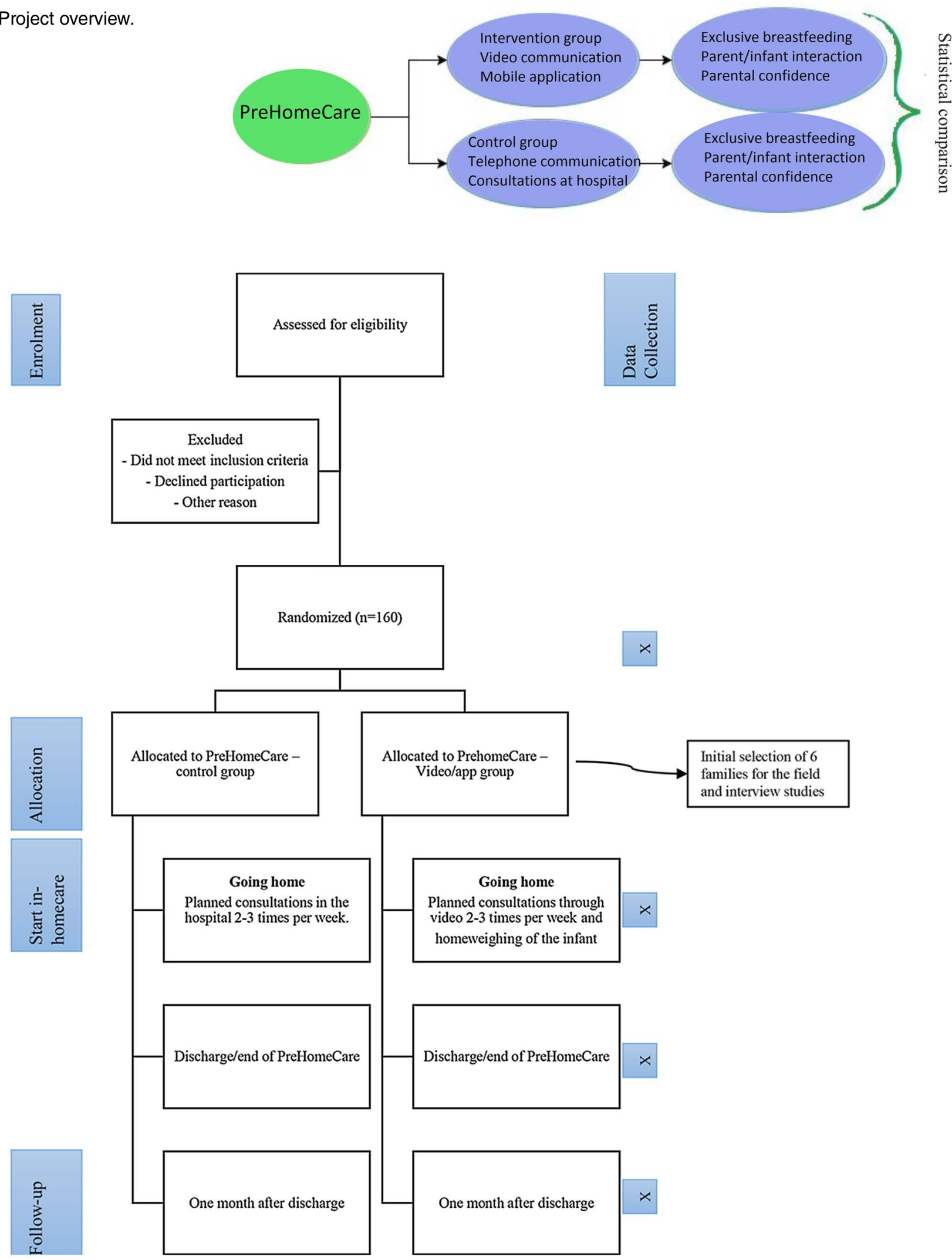

Figure 2 Flowchart of PreHome.

in the use of the smartphone application, video consultations and the manual. The remaining staffs at the neonatal wards have received training at staff meetings and bedside.
All the project nurses on the wards have quarterly meetings with the project sponsor/investigator to discuss study challenges, study progress, programme delivery and data collection. 


\section{Procedure}

The intervention group will receive the smartphone application at inclusion, the manual ${ }^{32}$ with instructions for its use and training in how to use the application in the hospital. When the families return home, they will use the application, attend the planned video consultations 2-3 times a week and have the ability to call the neonatal ward 24 hours a day, 7 days a week, until the infant receives full nutrition from the breast or bottle and gains weight (minimum 20-25 g/day), see figure 3 . Parents will borrow a baby scale to weigh the baby at home. All equipment (phone, weighting scale) will be provided from the neonatal department throughout the project.

The mobile application and video conference system, Præmatur Lifeline Viewcare A/S Herlev, ${ }^{33}$ were developed through clinical and parental evaluation and aim to provide the parents a secure and safe early inhomecare experience. The application addresses the parents' wish to have access to guidance around the clock and consists of three components: (1) usable knowledge (breast feeding, breast feeding position, infant signals, skin-to-skin contact, physiotherapy and so on), (2) video contact/participatory guidance, through which parents can contact the ward and (3) data registration (nutrition, vitamins and weight), including the ability to receive reminders, access patient history and share reports (notes, nutrition and weight) with the hospital. The digital solution activates knowledge through the search options and info icons included in the application.

The control group will be offered hospital consultations (usual care) 2-3 times a week (for 1-2 hours) and the opportunity to call the neonatal ward 24 hours a day, 7 days a week, until the infant receives full nutrition from the breast or bottle and gains weight (minimum 20-25 g/day). The parents will record the infant's nutrition between hospital visits on paper.

Both groups will learn to insert the feeding tube, receive training in first aid before PreHomeCare and will borrow breast pumps (if needed) as part of the PreHomeCare programme. All parents will receive leaflets and verbal information concerning the care of the infant. The overlap between the intervention and control groups while at the ward is not expected to have any implications on the results. If an infant requires
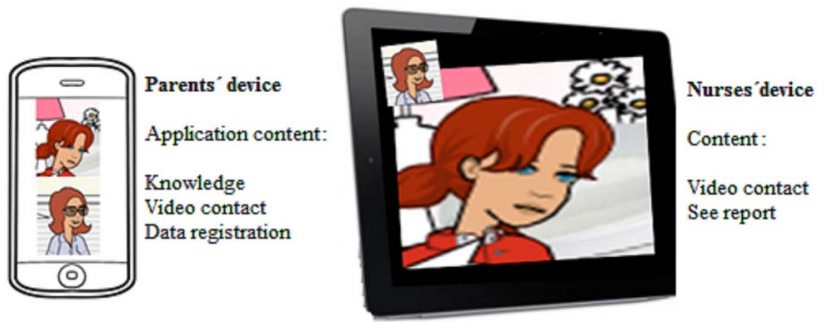

Figure 3 Parents' and nurses. medical and/or other services during the PreHomeCare programme, these services will be offered regardless of randomisation group.

\section{Power calculation and sample size}

The main hypothesis is that a larger proportion of breastfeeding women from the intervention group will continue breast feeding at least 1 month after discharge. Prior research ${ }^{6-9}$ suggests that the intervention will be able to increase the proportion of exclusively breastfed infants by providing easily accessible knowledge about breast feeding, guidance at all hours from a nurse and the ability to weigh the infant at home. Applying a significance level of $5 \%$ for a two-sided two sample proportion test, a sample size of 80 in each group would lead to a power of $80 \%$ to detect a difference of $55 \%$ versus $41.5 \%$ of breastfeeding women in the two groups after 4 weeks, assuming that $68 \%$ of mothers were breast feeding at discharge. ${ }^{8}$

Concerning the scores on the Mother and baby interaction scale (MABISC) and the Karitane parenting confidence scale (KPCS), ${ }^{34}$ small differences between two points in the scores can be detected with a power of $80 \%$ based on power calculations for a two sample t-test (data for the calculations are based on previous publications using the MABISC and KPCS). The inclusion period is expected to be 2 years based on the sample size calculations, a participation rate of 0.7 and a withdrawal rate of 0.1 , resulting in the inclusion of a minimum of 160 premature infants.

\section{Variables and data collection}

The primary outcome is the proportion of exclusive breastfeeding mothers 1 month after discharge/end of the PreHomeCare programme and is defined as infants feeding directly at/from the breast or receiving mother's expressed milk in a bottle based on the definition provided by the $\mathrm{WHO}^{36}$ (see table 1 ).

The secondary outcomes are parent-baby interactions according to the MABISC, ${ }^{35}$ parents' feeling of confidence determined by the $\mathrm{KPCS}^{34}$ and active knowledge of the premature infant 1 month after discharge/end of the PreHomeCare programme. The MABISC measures the mother-child relationship using 10 items scored on a five-point Likert scale (never, not often, occasionally, most of the time, always). The KPCS measures the parenting confidence of the infants' parents using 12 items rated on a 4-point Likert scale (no, hardly ever; no, not very often; yes, some of the time; yes, most of the time) (see table 1).

The secondary variables include data on the infant, parents' socio-demographic characteristics, breast feeding experience, experience with the PreHomeCare programme and number of contacts with the hospital after discharge (see table 1).

Data collection will be completed using a self-reported questionnaire that will be emailed to the mothers and completed electronically by the mother with assistance 
Table 1 Data collection

\begin{tabular}{|c|c|c|c|c|c|c|}
\hline Data collection & Baseline & $\begin{array}{l}\text { During } \\
\text { PreHomeCare }\end{array}$ & $\begin{array}{l}\text { End of } \\
\text { PreHomeCare/ } \\
\text { discharge }\end{array}$ & $\begin{array}{l}1 \text { month } \\
\text { after } \\
\text { discharge }\end{array}$ & $\begin{array}{l}\text { 1-year } \\
\text { follow-up }\end{array}$ & Source \\
\hline $\begin{array}{l}\text { Proportion of exclusive } \\
\text { breastfeeding mothers } \\
\text { Duration (days) of exclusive } \\
\text { breast feeding }\end{array}$ & & & $x$ & $x$ & $x$ & $\begin{array}{l}\text { Hospital record/ } \\
\text { Self-reported } \\
\text { questionnaire }\end{array}$ \\
\hline $\begin{array}{l}\text { MABISC, KPCS, parental } \\
\text { knowledge }\end{array}$ & $x$ & & $x$ & $x$ & $x$ & $\begin{array}{l}\text { Self-reported } \\
\text { questionnaire }\end{array}$ \\
\hline $\begin{array}{l}\text { Data on infant } \\
\text { Infant's weight and nutrition, } \\
\text { bottle and/or partial breast } \\
\text { feeding }\end{array}$ & $x$ & $x$ & $x$ & $x$ & $x$ & $\begin{array}{l}\text { Hospital record/ } \\
\text { Self-reported } \\
\text { questionnaire }\end{array}$ \\
\hline $\begin{array}{l}\text { Birth data (GA, weight, } \\
\text { length), diagnosis, date of } \\
\text { birth, gender, treatment } \\
\text { received in the hospital, date of } \\
\text { breast feeding/bottle feeding } \\
\text { initiation }\end{array}$ & $x$ & & & & & Hospital record \\
\hline $\begin{array}{l}\text { Socio-demographic data on } \\
\text { parents } \\
\text { Age, education, parity, income, } \\
\text { residence, marital and } \\
\text { cohabitation status, smoking, } \\
\text { mother's height and weight }\end{array}$ & $x$ & & & & & $\begin{array}{l}\text { Self-reported } \\
\text { questionnaire }\end{array}$ \\
\hline $\begin{array}{l}\text { Breast feeding experience } \\
\text { Previous breast feeding } \\
\text { experience, breastfeeding } \\
\text { self-efficacy (BSES-SF) } \\
\text { general health, perinatal } \\
\text { conditions, skin-to-skin contact } \\
\text { (hours per day) }\end{array}$ & $x$ & & $x$ & $x$ & & $\begin{array}{l}\text { Self-reported } \\
\text { questionnaire }\end{array}$ \\
\hline $\begin{array}{l}\text { Experience in the neonatal } \\
\text { ward } \\
\text { Experience with: the } \\
\text { preparation, the } \\
\text { implementation and the } \\
\text { termination of PreHomeCare } \\
\text { Intervention group: questions } \\
\text { about using the infant scale } \\
\text { video consultation and } \\
\text { smartphone application }\end{array}$ & & & $\mathrm{x}$ & $x$ & & $\begin{array}{l}\text { Self-reported } \\
\text { questionnaire }\end{array}$ \\
\hline $\begin{array}{l}\text { Number of contacts with the } \\
\text { hospital } \\
\text { Number of readmission(s), } \\
\text { time to readmission and } \\
\text { reason(s) for readmission }\end{array}$ & & & $x$ & $x$ & $x$ & $\begin{array}{l}\text { Hospital record/ } \\
\text { Self-reported } \\
\text { questionnaire }\end{array}$ \\
\hline $\begin{array}{l}\text { Field observations (intervention } \\
\text { group) }\end{array}$ & & $x$ & & & & \\
\hline Interviews (intervention group) & & & & $\mathrm{x}$ & & \\
\hline
\end{tabular}

from the father or another close relative using the online survey programme SurveyXact. ${ }^{37}$ Data will be collected equally in both groups. To minimise the number of those lost to follow-up, an email (and a text message) will be sent twice as a reminder. No gifts or cash incentives will be used to motivate participation.

Survey data will be obtained at four time points: inclusion, discharge/end of the PreHomeCare programme, 1 month after discharge and 1 year after discharge. The 
questionnaires will be distributed by the principal investigator.

The questionnaire contains $50-70$ items. The validity and reliability of the study will be enhanced by using validated questions. Questions will be added for topics in which we have not found validated items, namely, to evaluate the use of the equipment (scale, smartphone, application) (table 1). All questions including the selfconstructed ones were assessed for face validity. The selfreported questionnaire was pretested among 10 different parents to identify the duration of the questionnaire, any ambiguities and difficult or poorly worded questions by debriefing with the respondents; ${ }^{38}$ the questionnaire was then revised and finally tested again in two rounds.

Data on the infant will be gathered from hospital records and from application data by the nurses. These data will be registered in electronic case report forms (eCRFs) in an online Clinical Trial Management System $^{39}$ by the responsible nurses. The data collection time points are depicted in table 1.

\section{Statistical analysis}

Quantitative data will be analysed using STATA/IC V.14.0 (Stata Corp CP, Texas, USA) software. The reporting of results will follow the guidelines of the CONSORT statement. $^{31}$

The results of the intervention and control group will be compared and analysed following the intention to treat' principle and randomisation will remain intact. The study has a cluster structure with care in different hospitals, and this clustering will be considered when data are analysed (use of a random effects model is a possibility). Data will initially be analysed with univariate analysis using a $2 \times 2$ contingency table $\left(\chi^{2}\right.$ test and Fisher's exact test). Multivariate regression models will be used to adjust for confounders and other statistically significant explanatory variables. However, the initial randomisation should reduce the overall risk of confounding. The effect of the intervention according to the proportion of infants exclusively breast fed will be analysed using linear regression models. The MABISC and KPCS will be analysed using paired and unpaired t-tests. The number of readmissions will be evaluated using Poisson regression and will be further assessed by Kaplan-Meier curves as time to first readmission. A significance level of 0.05 will be applied in all analyses.

\section{Process evaluation study design}

Field observation study

The aim of this field study is to explore the nurses' communication (nursing practice, dialogue and information) through passive participant observation of the video consultations while in the parents' home. ${ }^{41}$ As the transition from hospital to home can be a vulnerable period for parents, they may require guidance. The field observation method was chosen to explore nursing practices and will be based on Spradley's methodology regarding 'participant observation'. ${ }^{41}$ The observations will occur in the parents' homes and will focus on the nurse's communication and interaction with the parents on screen to gain insight into how video communication affects their dialogue.

\section{Interview study}

A qualitative evaluation will be conducted to explore how parents of premature infants experience the PreHomeCare programme and the use of technology, that is, applications and video consultations, and the baby scale. As parents describe the transition from hospital to home as being difficult, and as they have reported feeling insecure, a qualitative interview study was chosen to gain in-depth knowledge of the parents' perspectives of PreHomeCare. The study will be planned and conducted with inspiration from Kvale and Brinkmann's methodology. ${ }^{42}$ The interview study will explore PreHomeCare from the parents' perspective by uncovering the meaning contained in their experiences.

\section{Data collection}

Six families will initially be selected from the intervention group to be the subjects of the field observations. In accordance with the qualitative tradition, the exact number of participants cannot be determined in advance. The data collection will continue until saturation has been reached, that is, until no new information is obtained. ${ }^{43}$ The selected parents will be informed about the aim of the study after randomisation. To ensure variation in the sample, the respondents will be strategically selected based on the premature infants' GA at birth (GA is related to the length of hospitalisation). The observations will occur in the parents' home during the three scheduled video consultations. The observer will stand to the side during the video consultations in the parents' home and will conduct an informal follow-up with the parents. Through 'passive participant observation' of the situation (video consultations), the people (parents, infants and nurse(s)) and the physical setting can be openly observed. Field notes will be recorded according to an observation guide inspired by the theory of 'active listening'. ${ }^{44}$ Thus, concrete descriptions of what is said and done, the tone of voice, the researcher's impressions and the assumptions of the communication between nurse(s) and parents will be documented. ${ }^{41}$ After the observations, the field notes will be written in prose.

Parents in the field observation study will be interviewed $^{45} \sim 1$ month after the end of the PreHomeCare programme, as the parents are expected to have reflected on the programme by this time point. As fathers and mothers may experience PreHomeCare differently, clarifying questions will be addressed to each parent using a semistructured interview guide. The interview will occur in the parents' home or in a place of the parent's preference. The primary aim of the interview will be to explore the main question: 'how did you experience PreHomeCare?' with a particular focus on 
what was especially meaningful for the parents. The interviewer will listen with an open mind and ask clarifying questions to gain as close an understanding as possible of the parents' everyday life experiences with PreHomeCare and the technology. Immediately after the interviews, the mood and other impressions will be noted, and the taped interviews ${ }^{42}$ will be transcribed verbatim.

\section{Data analysis of the qualitative substudies}

The transcripts of the interview data will be analysed using qualitative content analysis. ${ }^{46}$ This process includes open coding in which notes and headings are written in the text and are subsequently grouped into meaning units. Codes are compared with identify differences and similarities and are then transformed into categories and subcategories. Finally, the underlying meanings will be interpreted and formed into themes, either at the manifest or the latent level. Considering the first author's background in the neonatal ward, objectivity during data analysis will be gained through an awareness of the author's preunderstanding and through critical reflections to maintain an open mind throughout the analysis process. ${ }^{42}$ The supervisors, $\mathrm{AN}$ and $\mathrm{HK}$, will be involved in the analysis and interpretation of the data. The first author will do the primary coding. Subsequently, MHP, AN and HK will conduct the analysis and discuss the codes, emerging categories and subcategories with each other until consensus is reached.

\section{Ethical considerations, registration number and name of trial registry}

This project is registered with Clinicaltrials.gov; Clinical. gov ID NCT02581800.

Parents will be informed about the project verbally and in writing; the purpose of the study, the right to withdraw and the confidentiality of the data will be explained by project nurses, and informed consent will be obtained before inclusion. The parents will have a minimum of 1 day to decide whether they wish to participate. Confidentiality will be guaranteed, and participation is voluntary. The participants will be informed that the results of the interviews and field study will be included in publications. The data will be safely stored, and only MHP will have access to the final study data set. Positive as well as negative results of the effect (1) and process evaluation (2) will be published in separate manuscripts. The ethical aspects of the study follow the basic principles for research outlined in the Helsinki Declaration and the Northern Nurses' Federation (2003).

\section{Perspectives}

This study will lead to an improved understanding of the effect of the PreHomeCare programme and parents' experiences with it. Furthermore, it will provide insight into the factors needed to define clear goals regarding the quality of the treatment and care of premature infants in the end stages of their hospital admission/ during PreHomeCare. The knowledge gained from this project is expected to be used to further develop care for premature infants. The increased effectiveness of infant care can address challenges in healthcare and help redistribute economic and professional health service resources. The parents/infants who reside further away from the hospital could be able to use this technology for different hospital follow-up visits.

This study will contribute new knowledge of whether video consultations (participatory guidance) and Smartphone applications (usable knowledge and data registration) address parental needs for support during early inhomecare.

\section{Author affiliations}

${ }^{1}$ Department of Public Health, Section for Nursing, Aarhus, Denmark

${ }^{2}$ Department of Paediatrics, Næstved Hospital, Næstved, Denmark

${ }^{3}$ Faculty of Health Sciences, University College, Skejby, Denmark

${ }^{4}$ Medical Microbiology Department, Slagelse Hospital, Slagelse, Denmark

Acknowledgements We would like to thank the project nurses Birgit Hagelskær Dam (ICBLC), Catarina Krogh Andersen, Charlotte Maria Melgaard, Dorte Lissi Steen Hansen, Hanne Dalsgaard Loberg, Inge Nedergaard Henriksen, Inger Norup, Irene Dahlstrøm Larsen, Kirstine H Rotvig Erichsen, Merete van Deurs Petersen, Sille Nymann, Tina Thaulov Stoltenborg and Vibeke Fris Kyndesen (ICBLC) and all other staff at Næstved, Roskilde and Viborg neonatal departments for their daily work, engagement and involvement in the project. Furthermore, we would like to thank the nurse managers, Hanne Schjøning, Lis Dueholm, Birthe Kruuse, Karin Hallum (ICBLC) and Annemi Frandsen, the doctors, Gholamreza Krog Dayani, Hristo Stantchev and Jens Peter Nielsen, and the other ward staff for making the project possible and ensuring the organisational framework to support the project.

Contributors MHP conceived the study and drafted the project description and protocol under supervision from associate professors HK and AN. RD served as the statistical supervisor. HS contributed with comments on the study protocol and project description as well as by assuming medical responsibility. MHP obtained funding for this PhD project. All authors contributed to the final manuscript, and all authors can be held accountable for the accuracy and integrity of any part of the work. All authors have read and approved the final manuscript.

Funding This PhD project is financed by Aarhus University, the Danish foundation TrygFonden, the Health Foundation, Danish Nurses' Organization, Region Zealand Health Scientific Research Foundation and the local research foundation of NSR hospital.

Competing interests None declared.

Ethical approval This project has been approved by the Regional Ethics Committee (Region Zealand, Denmark), registration number: SJ-431, and the Danish Data Protection Agency, registration number: REG-113-2014.

Patient consent Obtained.

Provenance and peer review Not commissioned; externally peer reviewed.

Open Access This is an Open Access article distributed in accordance with the Creative Commons Attribution Non Commercial (CC BY-NC 4.0) license, which permits others to distribute, remix, adapt, build upon this work noncommercially, and license their derivative works on different terms, provided the original work is properly cited and the use is non-commercial. See: http:// creativecommons.org/licenses/by-nc/4.0/

\section{REFERENCES}

1. The partnership for maternal $\mathrm{NaCH}$, save the children; World Health Organization. Born too soon. The global action report on preterm birth. 2012. http://www.who.int/pmnch/media/news/2012/preterm birth_report/en/?utm_source=LifeSiteNews.com+Daily 
+Newsletter\&utm_campaign=05d7e64240-LifeSiteNews_com Canada_Full_Text 06_04_2012\&utm_medium=email (accessed 29 Jan 2016)

2. Broedsgaard A, Wagner L. How to facilitate parents and their premature infant for the transition home. Int Nurs Rev 2005;52:196-203

3. Sundhedsdatastyrelsen. Fødsler og fødte-Fødselsregisteret. http:// www.esundhed.dk/sundhedsregistre/MFR/Sider/F\%C3\% B8dselsregisteret.aspx (accessed 24 Sep 2016).

4. Boykova M, Kenner C. Transition from hospital to home for parents of preterm infants. J Perinat Neonatal Nurs 2012;26:81-7.

5. Zelkowitz $\mathrm{P}, \mathrm{Na} \mathrm{S}$, Wang $\mathrm{T}$, et al. Early maternal anxiety predicts cognitive and behavioural outcomes of VLBW children at 24 months corrected age. Acta Paediatr 2011;100:700-4.

6. Måstrup R. Breastfeeding of preterm infants. Associated factors in infants, mothers and clinical practice. Lunds Universitet, 2014.

7. Funkquist EL, Tuvemo T, Jonsson B, et al. Influence of test weighing before/after nursing on breastfeeding in preterm infants. Adv Neonatal Care 2010;10:33-9.

8. Maastrup R, Hansen BM, Kronborg $\mathrm{H}$, et al. Breastfeeding progression in preterm infants is influenced by factors in infants, mothers and clinical practice: the results of a national cohort study with high breastfeeding initiation rates. PLOS ONE 2014;9:e108208.

9. Renfrew MJ, McCormick FM, Wade A, et al. Support for healthy breastfeeding mothers with healthy term babies. Cochrane Database Syst Rev 2012:CD001141.

10. Brødsgaard A, Zimmermann R, Petersen M. A preterm lifeline: early discharge programme based on family-centred care. J Spec Pediatr Nurs 2015;20:232-43.

11. Ortenstrand A, Winbladh B, Nordström G, et al. Early discharge of preterm infants followed by domiciliary nursing care: parents anxiety, assessment of infant health and breastfeeding. Acta Paediatr 2001;90:1190-5.

12. Dellenmark-Blom $\mathrm{M}$, Wigert $\mathrm{H}$. Parents' experiences with neonatal home care following initial care in the neonatal intensive care unit: a phenomenological hermeneutical interview study. J Adv Nurs 2014;70:575-86.

13. Mai D, Børch K, Smidt L, et al. Tidligt hjemmeophold —et tværfagligt kvalitetsudviklingsprojekt. København: Neonatalklinikken,

Rigshospitalet, 2000

14. Ortenstrand A, Waldenström U, Winbladh B. Early discharge of preterm infants needing limited special care, followed by domiciliary nursing care. Acta Paediatr 1999;88:1024-30.

15. Bissell G, Wood A, Peak S, et al. Changing practice by the earlier introduction of tube feeding at home. Infant 2009;5:150.

16. Gund A, Sjöqvist BA, Wigert $H$, et al. A randomized controlled study about the use of eHealth in the home health care of premature infants. BMC Med Inform Decis Mak 2013;13:22.

17. Weis J. Guided family-centered care. Copenhagen University, 2014.

18. Fenwick J, Barclay L, Schmied V. Struggling to mother: a consequence of inhibitive nursing interactions in the neonata nursery. J Perinat Neonatal Nurs 2001;15:49-64.

19. Sáenz P, Cerdá M,, Diaz JL, et al. Psychological stress of parents of preterm infants enrolled in an early discharge programme from the neonatal intensive care unit: a prospective randomised trial. Arch Dis Child Fetal Neonatal Ed 2009;94:F98-104.

20. Collins CT, Makrides M, McPhee AJ. Early discharge with home support of gavage feeding for stable preterm infants who have not established full oral feeds. Cochrane Database Syst Rev 2003:(4): CD003743.

21. Lindberg B, Axelsson K, Ohrling K. Experience with videoconferencing between a neonatal unit and the families' home from the perspective of certified paediatric nurses. $J$ Telemed Telecare 2009:15:275-80.

22. Ahnfeldt AM, Stanchev $\mathrm{H}$, Jørgensen $\mathrm{HL}$, et al. Age and weight at final discharge from an early discharge programme for stable but tube-fed preterm infants. Acta Paediatr 2015;104:377-83.
23. Lopez GL, Anderson KH, Feutchinger J. Transition of premature infants from hospital to home life. Neonatal Netw 2012:31:207-14.

24. Robinson C, Gund A, Sjöqvist BA, et al. Using telemedicine in the care of newborn infants after discharge from a neonatal intensive care unit reduced the need of hospital visits. Acta Paediatr 2016;105:902-9.

25. Danbjørg DB, Wagner L, Kristensen BR, et al. Intervention among new parents followed up by an interview study exploring their experiences of telemedicine after early postnatal discharge. Midwifery 2015;31:574-81.

26. Garne K, Brødsgaard A, Zachariassen G, et al. Telemedicine in neonatal home care: identifying parental needs through participatory design. JMIR Res Protoc 2016;5:e100.

27. Currell $\mathrm{R}$, Urquhart $\mathrm{C}$, Wainwright $\mathrm{P}$, et al. Telemedicine versus face to face patient care: effects on professional practice and health care outcomes. Cochrane Database Syst Rev 2000:CD002098.

28. Epstein EG, Sherman J, Blackman A, et al. Testing the feasibility of Skype and FaceTime updates with parents in the neonatal intensive care unit. Am J Crit Care 2015;24:290-6.

29. Bartholomew L, Parzel G, Kok G. Planning health promotion programs. An intervention mapping approach. San Francisco, CA: Wiley Imprint, 2011

30. Stark AR, American Academy of Pediatrics, Committee on Fetus and Newborn. Levels of neonatal care. Pediatrics 2004;114: 1341-7.

31. Altman DG, Moher D. Declaration of transparency for each research article. BMJ 2013;347:f4796.

32. Hägi-Pedersen M-B. Præmatur appen PreHomeCare

brugervejledning. Næstved: Childrens Ward, 2015.

33. Sjælland S. Børne apps på rundtur. http:// sundhedsinnovationsjaelland.dk/content/borne-apps-pa-rundtur (accessed 3 Oct 2014)

34. Crncec R, Barnett B, Matthey S. Development of an instrument to assess perceived self-efficacy in the parents of infants. Res Nurs Health 2008;31:442-53.

35. Hoivik MS, Burkeland NA, Linaker OM, et al. The Mother and Baby Interaction Scale: a valid broadband instrument for efficient screening of postpartum interaction? A preliminary validation in a Norwegian community sample. Scand J Caring Sci 2013;27:733-9.

36. World Health Organization W. Exclusive breastfeeding. http://www. who.int/nutrition/topics/exclusive_breastfeeding/en/ (accessed 11 May 2016).

37. SurveyXact. SurveyXact. http://www.surveyxact.dk/

38. Fayers PM, Machin D. Quality of life - the assessment, analysis and reporting of patient-reported outcomes. 3rd edn. Chichester, UK: Wiley Blackwell, 2016.

39. Easytrial. Online Clinical Trial Management System. http://www. easytrial.net/en/\#sthash.Q8DsCAUJ.dpbs

40. Dennis CL. The breastfeeding self-efficacy scale: psychometric assessment of the short form. J Obstet Gynecol Neonatal Nurs 2003;32:734-44.

41. Spradley J. Participant observation. Belmont, CA: Wadsworth CENGAGE Learning, 1980

42. Kvale S, Brinkmann S. Interview- Introduktion til et håndværk. 2. udgave, 1. oplag ed. København: Hans Reitzels Forlag 2009.

43. Polit DF, Beck CT. Essentials of nursing research: appraising evidence for nursing practice. 8th edn. Philadelphia, PA: Wolters Kluwer Health /Lippincott Williams \& Wilkins, 2014.

44. Rogers C, Farson R. Active listening. Chicago, IL: Industrial Relations Center of The University of Chicago, 1957.

45. Mellor RM, Slaymaker E, Cleland J. Recognizing and overcoming challenges of couple interview research. Qual Health Res 2013:23:1399-407.

46. Graneheim UH, Lundman B. Qualitative content analysis in nursing research: concepts, procedures and measures to achieve trustworthiness. Nurse Educ Today 2004;24:105-12. 